\title{
Impaired negative feedback suppression of bile acid synthesis in mice lacking $\beta$ Klotho
}

\author{
Shinji Ito, ${ }^{1,2}$ Toshihiko Fujimori, ${ }^{1,3}$ Akiko Furuya, ${ }^{4}$ Junko Satoh, ${ }^{1}$ Yoko Nabeshima, ${ }^{1,3}$ \\ and Yo-ichi Nabeshima1,3
}

\begin{abstract}
1Department of Pathology and Tumor Biology, Graduate School of Medicine, and 2Horizontal Medical Research Organization, Faculty of Medicine, Kyoto University, Kyoto, Japan. ${ }^{3}$ Core Research for Evolutional Science and Technology (CREST), Japanese Science and Technology Corp., Saitama, Japan. ${ }^{4}$ Tokyo Research Laboratories, Kyowa Hakko Kogyo Co. Ltd., Tokyo, Japan.
\end{abstract}

\begin{abstract}
We have generated a line of mutant mouse that lacks $\beta$ Klotho, a protein that structurally resembles Klotho. The synthesis and excretion of bile acids were found to be dramatically elevated in these mutants, and the expression of 2 key bile acid synthase genes, cholesterol $7 \alpha$-hydroxylase (Cyp7a1) and sterol 12 $\alpha$-hydroxylase (Cyp8b1), was strongly upregulated. Nuclear receptor pathways and the enterohepatic circulation, which regulates bile acid synthesis, seemed to be largely intact; however, bile acid-dependent induction of the small heterodimer partner (SHP) NR0B2, a common negative regulator of Cyp7a1 and Cyp8b1, was significantly attenuated. The expression of $C y p 7 a 1$ and $C y p 8 b 1$ is known to be repressed by dietary bile acids via both SHP-dependent and -independent regulations. Interestingly, the suppression of $C y p 7 a 1$ expression by dietary bile acids was impaired, whereas that of $C y p 8 b 1$ expression was not substantially altered in $\beta k l o t h o^{-/-}$mice. Therefore, $\beta \mathrm{Klotho}$ may stand as a novel contributor to $C y p 7 a 1$-selective regulation. Additionally, $\beta$ Klotho-knockout mice exhibit resistance to gallstone formation, which suggests the potential future clinical relevance of the $\beta$ Klotho system.
\end{abstract}

\section{Introduction}

We previously reported the identification of a mouse gene, $\beta k$ lotho, that encodes a protein that structurally resembles Klotho (1). Deficiency of the Klotho protein results in various human aging-like phenotypes in mouse, including calcium homeostasis disorders (2). Both Klotho and $\beta$ Klotho are type I membrane proteins, which contain 2 family 1 glycosidase-like domains. They lack the characteristic glutamic acid residues essential for enzymatic activities and thus form a distinct subfamily within glycosidase family $1(3,4)$. $\beta$ klotho is predominantly expressed in the liver, pancreas, and adipose tissues (1). With the exception of mRNA expression profiles, not much regarding the molecular function of $\beta$ Klotho is known. We therefore generated $\beta k_{l o t h o}{ }^{-/}$mice. The gross appearance of knockout mice was normal upon initial examination. Interestingly and unexpectedly, however, bile acid metabolism was altered in these mice.

Bile acids are synthesized from cholesterol in the liver. They are vitally important for the intestinal absorption of lipids and lipid-soluble materials. In addition, bile acid biosynthesis is the sole pathway for the elimination of superfluous cholesterol. On the other hand, hydrophobic secondary bile acids generated by intestinal flora have toxic effects, leading to cholestasis and carcinogenesis $(5,6)$. Therefore, proper control of bile acid synthesis is crucial for animals. The rate-limiting step for the major bile acid synthetic pathway, which is catalyzed by cholesterol $7 \alpha$-hydroxylase (CYP7A1), is thus strictly regulated by numerous factors, with negative feedback regulated by bile acids representing the principal

Nonstandard abbreviations used: $\mathrm{CA}$, cholic acid; CDCA, chenodeoxycholic acid; CYP7A1, cholesterol $7 \alpha$-hydroxylase; CYP8B1, sterol $12 \alpha$-hydroxylase; EGFP, enhanced GFP; Fgfr4, fibroblast growth factor receptor 4; FXR, farnesoid X receptor; Himgr, 3-bydroxy-3-methylglutaryl coenzyme A reductase. LXR $\alpha$, liver X receptor $\alpha$; SHP, small heterodimer partner.

Conflict of interest: The authors have declared that no conflict of interest exists.

Citation for this article: J. Clin. Invest. 115:2202-2208 (2005).

doi:10.1172/JCI23076. one $(7,8)$. Nuclear receptors, kinases, and growth factor-mediated pathways have been demonstrated to be involved in this negative feedback regulation $(6,7,9-16)$. After conjugation in hepatocytes, bile acids are secreted, reabsorbed from intestine, and delivered back to the liver. Transportation across the membrane is carried out by a number of solute carrier proteins and $A B C$ transporters $(17,18)$. The intactness of this enterohepatic circulation is necessary for the appropriate regulation of bile acid metabolism.

Through the analysis of expression profiles of genes regulating bile acid synthesis, we propose potential roles for $\beta$ Klotho in negative regulatory pathways.

\section{Results}

Generation of the $\beta k l o t h 0^{-/-}$mouse. The mouse $\beta$ klotho locus consists of at least 8 exons. We have constructed a gene-targeting vector to delete most of the putative exon 1 that includes the first ATG codon via homologous recombination in ES cells (Figure 1A). Successful distuption of the $\beta$ klotho locus was confirmed by genomic Southern blot analyses using a probe flanking the region upstream of the targeting vector (Figure 1, A and B). Elimination of the $\beta$ klotho mRNA expression was confirmed by Northern blotting (Figure 1C) and further verified by RT-PCR using several primer pairs covering different regions of the remaining exons (data not shown). The absence of protein expression was also affirmed by Western blotting using a monoclonal antibody that recognizes the C-terminal portion of the second internal repeat ( $\beta$ KL2) of the $\beta$ Klotho protein (Figure 1D). Although we could detect transcripts for enhanced GFP (EGFP) with the same tissue distribution as those for $\beta k l o t h o$, we could not detect EGFP protein by Western blotting (data not shown).

$\beta k l o t h o^{-/-}$mice were viable, fertile, and appeared grossly normal under standard conditions. The number of the homozygous mice obtained was less than would be expected according to Mendelian inheritance laws (297:624:193, $\beta$ klotho $^{+/+} / \beta k_{\text {lotho }}{ }^{+/-} /$ $\left.\beta k l o t h 0^{--}\right)$. The body weights of homozygous animals were slightly 
A

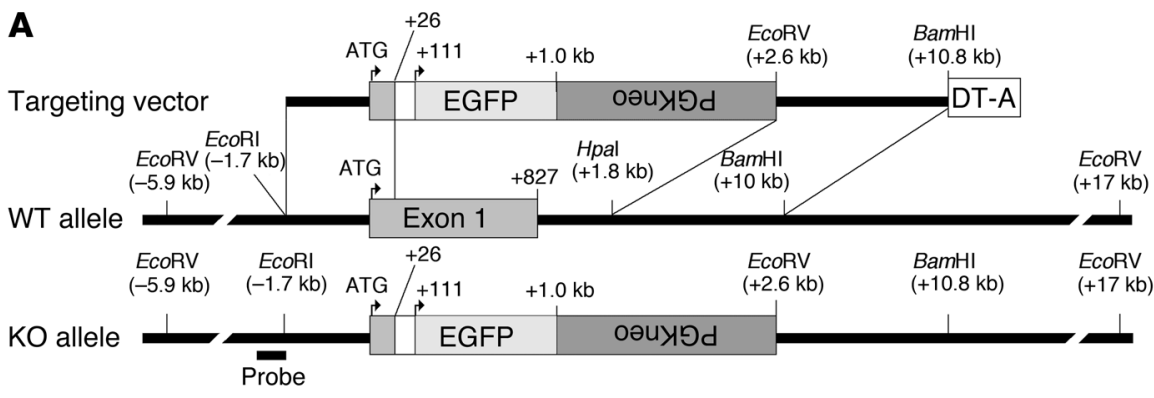

B

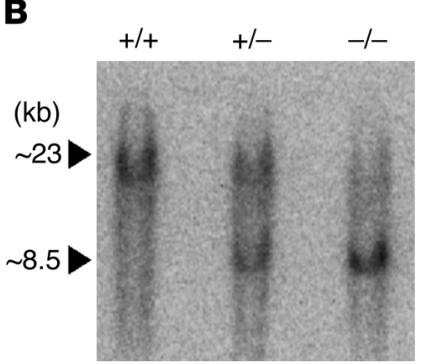

E

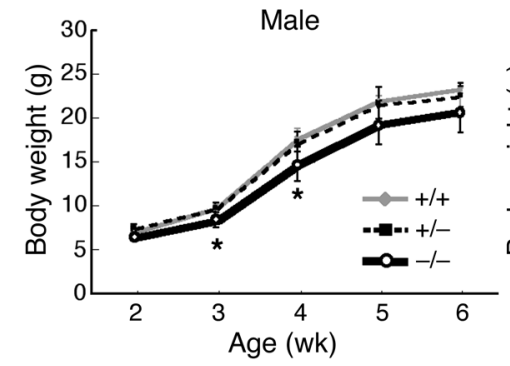

C

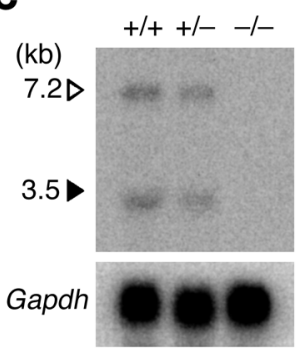

D Liver WAT Kidney
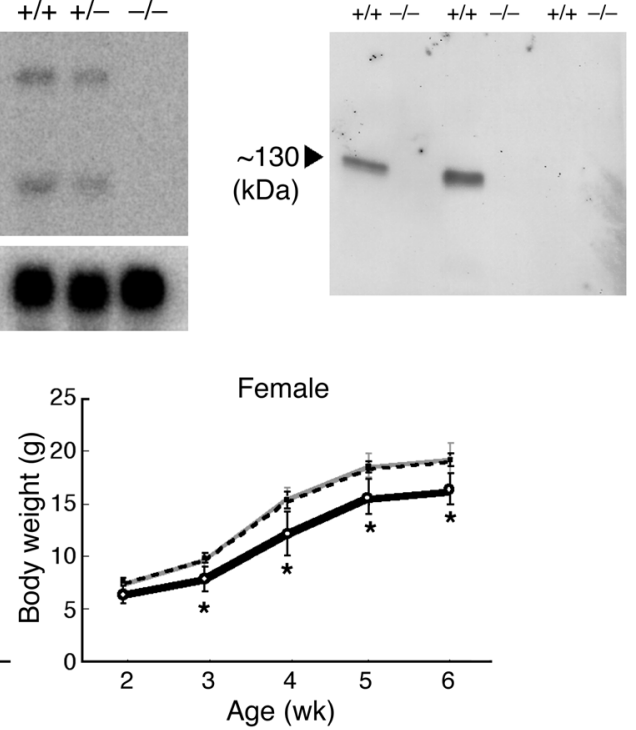

Figure 1

Targeted disruption of the $\beta k$ lotho gene. (A) Strategy for disruption of mouse $\beta$ klotho locus. Schematic diagrams of the targeting vector (top), wild-type allele (middle), and disrupted allele (bottom) are shown. Numbers correspond to the distance (bp) from the putative translation initiation site. The probe used for Southern blot analysis is shown at the bottom. (B) Southern blot analysis of EcoRV-digested genomic DNA. Blots were hybridized with the probe indicated in A. (C) Northern blot analysis of $\beta k$ lotho gene expression in the liver. The $\beta$ klotho-specific probe hybridizes with 2 species of transcripts (1). (D) Western blot analysis of $\beta$ Klotho protein expression. Total proteins $(15 \mu \mathrm{g})$ extracted from indicated tissues were blotted on a PVDF membrane and incubated with a $\beta$ Klothospecific monoclonal antibody. $\beta$ Klotho protein was detected only in $\beta$ klotho-expressing tissues of wild-type mice and was absent in tissues of $\beta k l o t h o^{-1-}$ mice. WAT, white adipose tissue. (E) Growth curve comparison of $\beta k l o t h o^{+/+}, \beta k l o t h o^{+/-}$, and $\beta k^{\prime}$ tho $^{-/-}$mice. Body weights were traced in mice from ages 2 to 6 weeks. Five to 12 mice for each group were examined. Error bars indicate SD. ${ }^{*} P<0.05$. but significantly reduced compared with those of wild-type and heterozygous littermates (Figure 1E). The decrease in body weight could not be attributed to the differences in the size of the liver or gonadal fat. The slight reduction in body weight was reproducibly seen in groups that were backcrossed 5 times into the C57BL/6 background (data not shown). Sections of various tissues including $\beta$ klotho-expressing tissues stained with $\mathrm{H} \& \mathrm{E}$ were carefully examined; however, histological differences between $\beta k \mathrm{klotho}^{-/-}$and wild-type mice could not be found. Serum markers relevant to $\beta$ klotho-expressing tissues such as serum triglyceride, fatty acids, cholesterol, phospholipid levels and enzyme activities derived from liver and pancreas were not significantly different between genotypes (data not shown).

Elevation of the synthesis and excretion of bile acids. $\beta$ klotho-expressing tissues are all involved in lipid metabolism; therefore, we first speculated that this molecule might be involved in some aspects of lipid metabolism (1). However, serum markers indicative of lipid metabolism were not significantly altered in $\beta k l o t h o^{-/-}$mice. We observed a slight reduction in serum cholesterol levels, although it did not reach statistical significance in mice of either sex (Figure 2A). This led us to focus on cholesterol and its metabolism in $\beta \mathrm{klotho}^{-/-}$mice. Since $\beta$ klotho is strongly expressed in the liver, where cholesterol is converted to bile acids (1), we examined the metabolism of these sterols in $\beta k$ lotho $^{-/-}$mice. We found that fecal bile acid excretion was dramatically elevated in the mutants compared with wild-type mice (Figure 2B). Daily food intake and total fecal amounts were similar in mice of both genotypes (Figure 2C), but the bile acid pool size was slightly increased in $\beta \mathrm{klotho}^{-/-}$mice, although the increase was statistically significant only in males (Figure 2D).

CYP7A1 is the most important enzyme for bile acid production, as it catalyzes the first and rate-limiting step of the classical bile acid synthetic pathway (8). We found that the expression of Cyp7a1 was dramatically elevated in $\beta k l o t h o^{-/-}$mice compared with that in the wild-type littermates (Figure 2E). More than 20 littermate pairs were examined, and the elevation was evident in all cases. It was also the case in mice that had been backcrossed 5 times into the C57BL/ 6 background (data not shown). Another enzyme, sterol $12 \alpha$-hydroxylase (CYP8B1), is also of importance, as it is indispensable for generation of the most abundant primary bile acid, cholic acid (CA) $(8,19)$. We examined the expression of Cyp8b1 and found that it was also strongly upregulated in $\beta k l o t h o^{-/-}$mice (Figure $2 \mathrm{E}$ ).

It is known that interruption of the enterohepatic circulation elicits the elevation of bile acid synthesis $(20,21)$. For instance, cholestyramine, an inhibitor of intestinal bile acid reabsorption, dramatically increases bile acid synthesis and excretion (21). We examined the state of the circulation. The mRNA expression of intestinal bile acid binding protein (I-babp), which is known to be induced in response to the absorption of bile acids (22), was considerably elevated in the small intestine of the $\beta k l_{\text {lotho }} /-$ mice (Figure 2F). This probably reflects the increased secretion of bile acids into the duodenum, and it is likely that bile acid absorption is largely intact in $\beta k$ lotho $^{-/-}$mice. Consistent genotype-dependent differences in the mRNA expression of hepatic canalicular transporters $\mathrm{Na}^{+}$-taurocholate-cotransporting protein 1 (Ntcp1; 

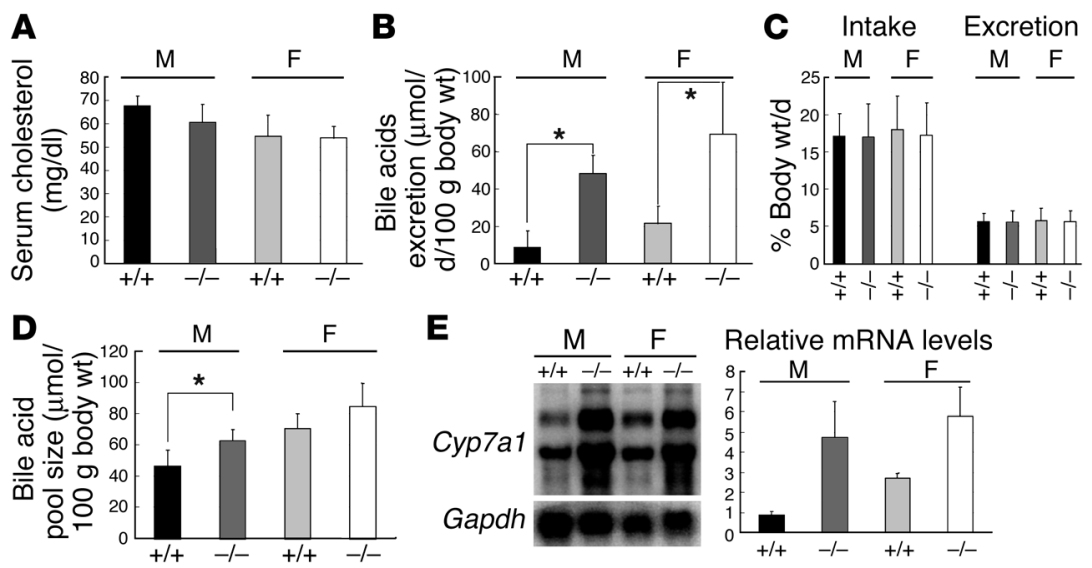

$\mathbf{E}$
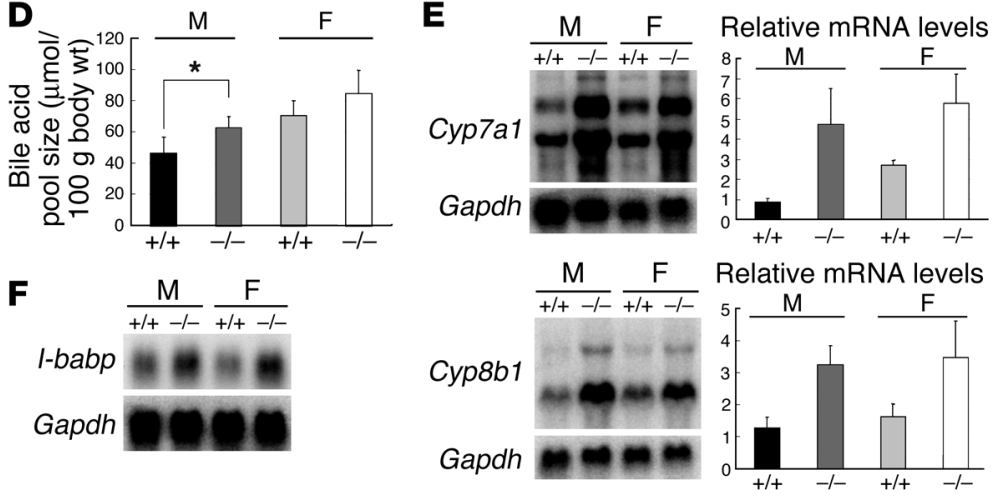

Figure 2

Altered bile acid metabolism in $\beta k l o t h o^{-/-}$mice. Graphs show the comparison of several parameters in wild-type and knockout mice. (A) Serum cholesterol levels were compared using 4-6 animals per group. (B) Stool samples were collected daily for 3 days from 4-6 mice per group. Fecal excretion of total bile acids was measured, and the averages of 12-18 samples per group are shown. ${ }^{*} P<0.05$. (C) Daily food intake and fecal excretion of 4 individuals per each genotype were examined for 3 days. (D) Comparison of bile acid pool size. Four to 10 mice per group were examined. (E) Expression of bile acid synthase genes Cyp7a1 and Cyp8b1 in wild-type and $\beta k l o t h o^{-/}$mice. Total RNA (30 $\left.\mu \mathrm{g}\right)$ from the livers of each genotype were analyzed. Representative results from 2 independent experiments are shown. Northern blots are shown in the left panel, and relative expression levels normalized for Gapdh levels are presented in the right panels. (F) Expression of intestinal bile acid binding protein (I-babp). Total RNA $(30 \mu \mathrm{g})$ from the whole small intestine was analyzed. Results shown are from samples taken from age- and sex-matched littermates. All data in this figure were derived from 7-week-old mice fed standard diets. M, male; F, female. Error bars indicate SD.

Slc10a1) and organic anion-transporting polypeptide 1 (Oatp1; Slc27a) and apical transporters bile acid-exporting pump (Bsep; $\mathrm{Abcb} 11)$ and multidrug resistance-associated protein 2 (Mrp2; Abcc2) were not observed. Also, the expression of ileal apical sodium-dependent bile acid transporter (Asbt; Slc10a2) was not reduced in $\beta k l o t h o^{-/-}$mice (data not shown). Additionally, the bile acid pool size was increased in $\beta k l o t h o^{-/}$mice, whereas it is reduced in enterohepatic circulation-deficient mice (20). These results led us to conclude that the enterohepatic circulation of bile acids is not significantly impaired in $\beta$ klotho ${ }^{-/}$mice.

Resistance to cholesterol gallstone formation. It has been reported that C57BL/6 mice develop cholesterol gallstones when cholesterol and bile acid-containing atherogenic (lithogenic) diets are administered (23). The formation is more severe in males than females and can be prevented by the overexpression of rat CYP7A1 in mice (23). We examined the response of $\beta k l o t h o^{-/}$mice to long-term administration of the atherogenic diet, which contains $1 \%$ cholesterol and $0.5 \%$ CA. Consistent with previous reports, wild-type mice developed severe gallstones after being fed the atherogenic diet for 8 weeks (Figure 3A, left). In contrast, gallstone formation was not observed in $\mathrm{Bklotho}^{-/-}$mice of either sex (Figure 3A, right). More than 14 mice for each genotype were examined, and no exceptions were seen. We also observed that the size of the gallbladder was smaller in $\beta k l o t h o^{-/-}$mice compared with that in wild-type mice of both sexes (Figure $3 \mathrm{~A}$ arrows).

When hypersaturated in bile, cholesterols crystallize to form gallstones. Many factors are known to be involved in the initial steps of gallstone formation (24). However, the most important factor for gallstone formation is excessive cholesterol relative to bile acids and phospholipids $(25,26)$. Since rat CYP7A1-overexpressing mice exhibit resistance to gallstone formation (23), increased bile acid synthesis appears to be sufficient for its prevention. This is also supported by the fact that the long-term administration of ursodeoxycholic acid solubilizes gallstones in bile (27).

To further investigate the mechanisms underlying the gallstone resistance, we examined hepatic, fecal, and serum cholesterol levels. Under normal feeding conditions, hepatic, fecal, and serum cholesterol levels were not significantly different between genotypes (Figures 3 , $\mathrm{B}$ and $\mathrm{C}$, left, and Figure 2A). When mice were challenged with an atherogenic diet, these parameters were dramatically elevated in both genotypes; however, we could not find any genotype-dependent differences common to both sexes (Figures 3, B and C, right, and Figure 3D). In both standard and atherogenic diet-fed conditions, we examined the expression of hepatic free cholesterol transporters $A b c g 5$ and $A b c g 8$ (also called sterolin-1 and sterolin-2), which are responsible for the secretion of free cholesterols into bile (28). The atherogenic diet dramatically elevated their expression; however, we could not find any overt genotype-dependent differences in either dietary condition (data not shown). Together, the results suggest that the gross cholesterol macrodynamics in the liver are not significantly altered in $\beta k l o t h o^{-/-}$mice. These observations suggested that gallstone resistance in $\beta k l o t h o^{-/-}$ mice resulted from the overproduction and secretion of bile acids.

Because hepatic and serum cholesterol levels were similarly elevated by the atherogenic diet in both genotypes, it was suggested that $\beta$ Klotho is not a dominant factor for gross cholesterol metabolism in the liver. It might play a rather limited role that is specific to the suppression of bile acid synthesis. However, since loss of $\beta$ Klotho did cause a slight decrease in serum cholesterol levels, it might be an important regulator for long-term sustainment of cholesterol homeostasis, at least under standard dietary conditions, despite its mild effects.

Gene expression profiles under basal and high bile acid dietary conditions. Gene expression profiles in the liver were examined in order to identify genes with distinct genotype-specific expression. The expression of more than 30 genes encoding nuclear receptors, transporters, P-450s and other enzymes, lipoprotein receptors, and apolipoproteins related to lipid, steroid, and xenobiotic metabolism were compared between wild-type and $\beta k l o t h o^{-/}$genotypes by Northern blotting in sex-matched littermate pairs. In addition to the 2 bile acid synthase genes already described, we 
A
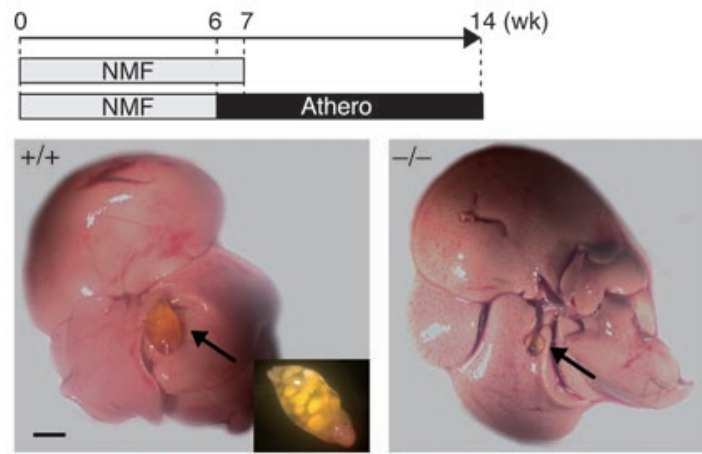

$+/+$
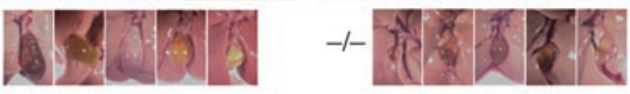

B

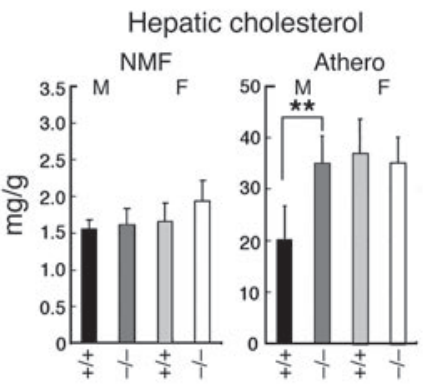

C

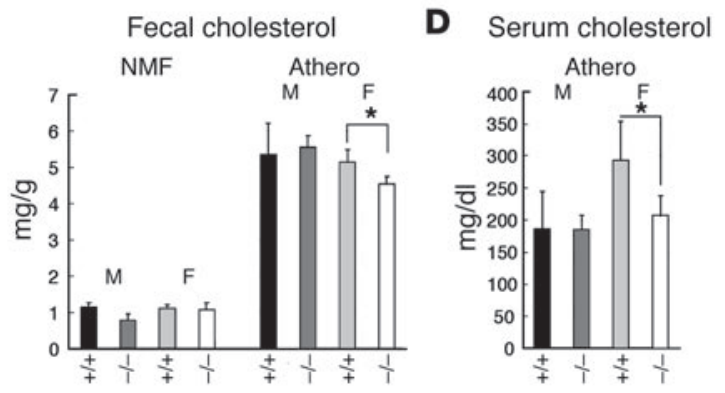

observed distinct genotype-dependent difference in the expression of 3-hydroxy-3-methylghtutaryl coenzyme A reductase (Hmgr), the rate-limiting enzyme for cholesterol biosynthesis (29). The expression of $\mathrm{Hmgr}$ was also strongly upregulated in $\beta \mathrm{klotho}^{-/-}$mice, in both males and females (Figure 4A).

Since bile acid metabolism was significantly altered in $\beta k l o t h o^{-/-}$ mice, likely as a result of the upregulation of the bile acid synthase genes, we examined the effect of dietary bile acids on the expression of Cyp7a1, Cyp8b1, and regulatory factors for these genes. Mice were fed the basal diet from the lactation period with their mothers up to 6 weeks of age. After that, the same diet or bile acid-containing derivatives of the basal diet were administered for an additional week. Two kinds of primary bile acids, CA and chenodeoxycholic acid (CDCA), were used at the concentrations of $1 \%$ (Figure $4 \mathrm{~B}$, top).

In wild-type animals, the expression of Cyp7a1 was detected when they were fed the basal diet and was completely diminished under administration of diets that contained bile acids. In $\beta k l o t h o^{-/-}$mice, Cyp7a1 expression was dramatically elevated compared with that of wild-type mice under the basal conditions. After dietary administration of bile acids, the expression levels were reduced; however, a significant portion of expression could not be eliminated (Figure 4B). In clear contrast, Cyp 861 expression was almost completely

\section{Figure 3}

Resistance of $\beta \mathrm{klotho}^{-/-}$mice to gallstone formation. (A) Cholesterol gallstones formed in wild-type mice (left) but not in $\beta k^{\prime} l o t h o^{-/}$mice on the atherogenic diet (Athero) (right). Diet administration regimens are shown at the top. Mice were sacrificed at the end of each regimen. Gallbladder is indicated (arrows). A magnified image of a wildtype gallbladder with severe gallstones is shown in the inset in the left panel. Examples of gallbladders from other individuals are shown below. Scale bars: $5 \mathrm{~mm}$. (B) The average hepatic cholesterol levels of 7-week-old standard diet-fed mice (NMF; left panel) and that of 14-week-old mice on the atherogenic regimen (right panel). Four to 7 mice per group were examined. (C) Average fecal cholesterol concentrations. Stool samples were collected daily for 3 days from 7 -week-old standard diet-fed mice (left) and 14-week-old mice on the atherogenic regimen (right). Three to 4 mice per group were examined. Results for stool samples from the same individuals were combined for the analyses. (D) Serum cholesterol levels of 14-week-old mice on the atherogenic regimen. Four to 7 mice per group were examined. Error bars indicate SD. ${ }^{\star} P<0.05 ;{ }^{* \star} P<0.005$.

diminished after dietary administration of bile acids, irrespective of genotype (Figure 4B). The expression levels were slightly higher in $\beta k l o t h 0^{-/-}$mice after the administration; however, a statistically significant difference between genotypes was not observed (Figure 4B). The expression levels of Hmgr under high bile acid conditions were not significantly different between genotypes (data not shown).

Since it is known that the expression of Cyp7a1 and Cyp $8 b 1$ is strongly regulated by the regulatory cascade that is mediated through farnesoid X receptor (FXR; NR1H4) and SHP, the expression of $S h p$ was examined in both basal and high bile acid states $(9$, 30). The expression level of Shp was slightly lower in the mutant, but the difference was not statistically significant (Figure 4B). After the administration of bile acid-enriched diet, the expression levels of Shp were significantly elevated in both mutant and wildtype mice. However, although the expression was dramatically increased in the wild-type mice (2.48- and 2.46-fold for CA and CDCA, respectively), the increase was rather weak in the mutant (1.64- and 1.79-fold for CA and CDCA, respectively) (Figure 4B). The expression profiles of Fxr did not differ significantly between genotypes, and those of $\beta$ klotho did not differ among the various dietary conditions (data not shown). Similar experiments were also performed with NMF (a standard diet) and NMF supplemented with $1 \% \mathrm{CA}$, although the animals were not age-matched in these experiments. Identical results were also obtained from these alternative experiments (data not shown).

\section{Discussion}

In order to define the molecular function of $\beta$ Klotho, we generated a $\beta k l o t h o^{-/-}$mutant mouse. $\beta k l o t h o^{-/-}$mice appeared grossly normal, although a slight reduction in body weight compared with wild-type and heterozygous mice was noted (Figure 1E). Hepatic, fecal, and serum cholesterol levels were not significantly altered in $\beta k l o t h o^{-/}$mice. Unexpectedly, however, we found that bile acid synthesis in the liver and its fecal excretion were dramatically enhanced in these mutants (Figure 2). Additionally, $\beta k l o t h o^{-/}$mice exhibited resistance to the formation of cholesterol gallstones when they were fed an atherogenic diet (Figure 3A). These phenotypes are likely to have resulted from the impaired negative feedback suppression of the bile acid synthesis.

It is believed that one of the major negative feedback pathways for bile acid production is mediated by nuclear receptors, 
A

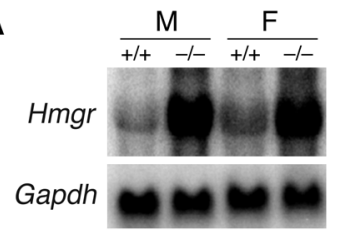

B
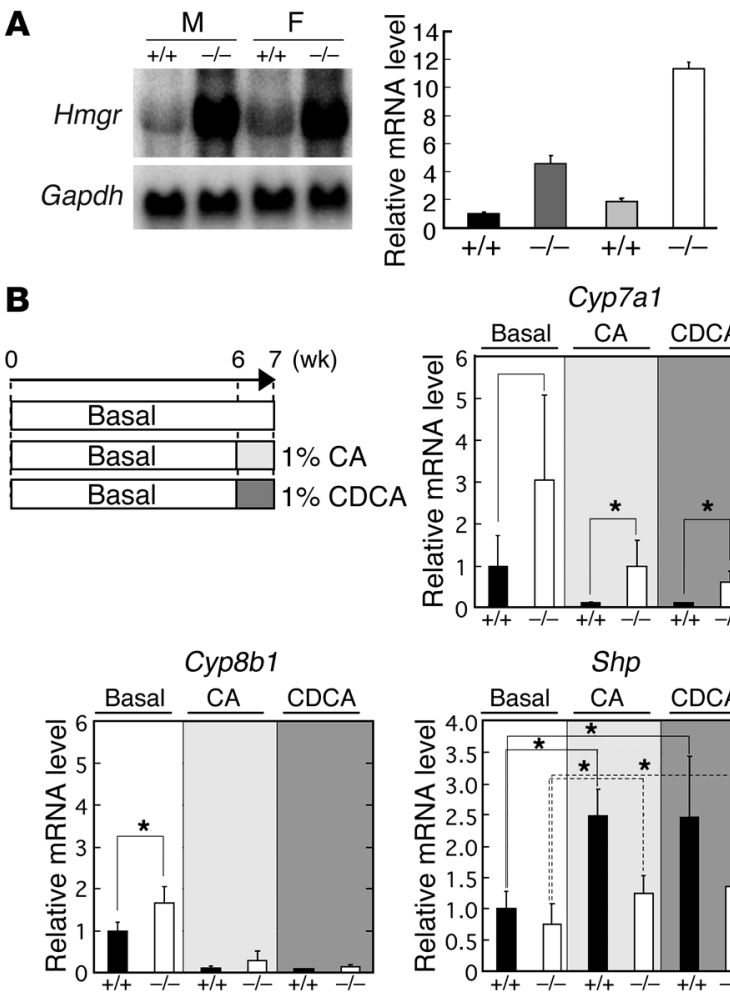
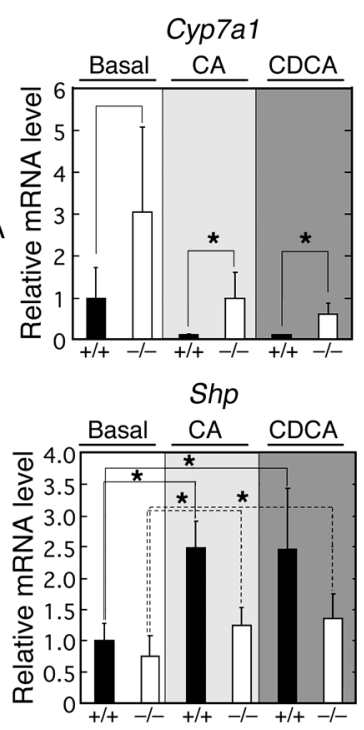

Figure 4

Gene expression profiles under various dietary conditions. (A) $\mathrm{Hmgr}$ mRNA expression in wild-type and $\beta k l o t h o^{-/-}$mice. Total RNA $(30 \mu \mathrm{g})$ from the liver was analyzed. Representative examples of 2 independent trials are shown. Northern blots are shown at the left, and relative expression levels compensated for Gapdh are shown in the right panel. (B) Relative expression levels of selected genes. The expression levels of Gapdh were used as internal controls. Relative expression levels of each gene were further standardized to the mean expression levels of wild-type animals fed the basal diet. The dietary regimens used for the examination of the effect of dietary bile acids are shown at the top. Mice were sacrificed at the end of each regimen. Effects of dietary $\mathrm{CA}$ and CDCA on the expression of Cyp7a1, Cyp8b1, and Shp were examined. Total RNA $(30 \mu \mathrm{g})$ from the liver was subjected to Northern blot analysis. The mean values obtained from 6 independent animals for each group are shown. Error bars indicate SD. ${ }^{*} P<0.05$.

including FXR and SHP. The bile acid receptor FXR induces SHP expression to suppress bile acid synthase gene expressions (30-32). In $\beta k l o t h o^{-/-}$mice, bile acid-mediated induction of Shp was significantly attenuated (Figure 4B). This limited blunting would be expected to contribute to the abnormal regulation of bile acid synthesis at least in a part. $\beta$ Klotho should participate in some aspects of the FXR/SHP cascade. On the other hand, other FXR target genes, I-babp (Figure 2F) and Fgf15 (data not shown), were appropriately upregulated in the intestine, responding to the increased bile acid influx. Therefore, FXR function seems to be only impaired in the induction of Shp in the liver.

Since bile acid-dependent suppression of bile acid synthase genes Cyp7a1 and Cyp8b1 is virtually intact in $\operatorname{Shp}^{-/-}$mice $(33,34)$, the expression of these genes must be diminished by bile acids, even though FXR/SHP cascade is impaired. In $\beta k l o t h o^{-/-}$mice, however, Cyp8b1 expression was completely suppressed, whereas that of Cyp7a1 was not. Therefore, $\beta$ Klotho should be involved not only in

the FXR/SHP cascade but also in a Cyp7a1-specific regulation that is independent of SHP. SHP-independent divergent regulation of Cyp $7 a$ and Cyp 861 was also demonstrated in $S h p^{-/-}$mice chronically treated with bile acids (35).

Pathways mediated via pregnane $\mathrm{X}$ receptor (NRII2) and PPAR $\alpha$ $(\mathrm{NRlCl})$ are possible candidates for SHP-independent redundant regulation. In addition to their authentic target genes, these nuclear receptors also suppresses the expression of Cyp7a1 $(6,10,11)$. We found that the expression levels of these receptors and their authentic target genes did not vary between genotypes (data not shown). Thus, these nuclear receptor-mediated pathways are likely to be largely functional in $\beta k$ lotho $o^{-/}$mice.

FGF-mediated regulation is also a potential candidate for SHPindependent regulation. Lack of fibroblast grow th factor receptor 4 (Fgfr4) elicits enhanced expression of Cyp7a1 in mice (15), and exogenous expression of FGF19, a specific ligand for FGFR4, suppresses Cyp7a1 expression (16). In human hepatocytes, FGF19 is induced by bile acids and suppresses CYP7A1 expression regardless of SHP expression levels (16). In addition, Shp expression levels decreased in constitutively activated human FGFR4-transgenic mice along with the levels of bile acid pools but did not increase in response to elevated bile acid levels in FGFR4-deficient mice (36). These results suggests that FGFR4mediated repression of Cyp7a1 is likely to be independent of SHP expression levels (36). Interestingly, there are several common phenotypes between $\beta k l o t h o^{-/-}$and $\mathrm{Fg}_{\mathrm{fr} \mathrm{H}^{-/-}}$mice. To our knowledge, simultaneous induction of these genes has not been found except in $\beta k_{l o t h o} o^{--}$and $\mathrm{Fg} f \mathrm{r}^{-/-}$mice. Additionally, $\beta \mathrm{klotho}^{-/-}$and Fgfr $4^{-/-}$mice similarly exhibit a small gallbladder size (15).

Other candidates for SHP-independent pathways are those mediated via JNK. In rat hepatocytes, bile acids elicit JNK activation concurrent with suppression of Cyp7a1 expression (13). Since JNK activation leads to the induction of SHP in these cells, JNKmediated regulation of Cyp7a1 probably involves SHP at least in a part (13). However, Cyp7a1 expression was enhanced even in the Shp-l- hepatocytes when treated with JNK inhibitor. Therefore, not all downstream targets of JNK signals are mediated through SHP (33). Notably, it has been demonstrated that FGFR4 is involved in JNK-signaling pathways, which suggests that these pathways are related to each other at least to some extent $(15,16,36)$.

The expression of Cyp7a1 is also under the strong feedforward regulation of cholesterol via liver X receptor $\alpha$ (LXR $\alpha$; NR1H3) (37). However, that is generally hypostatic to the negative feedback regulation (38). We were unable to precisely evaluate its effect; however, it is likely that LXR $\alpha$-mediated regulation by cholesterol is largely intact in the $\beta \mathrm{klotho}^{-/}$mouse, as the expression of other LXR $\alpha$ target genes, $A b c g 5$ and $A b c g 8$ (39), seems to be normally induced by the elevation of hepatic cholesterol levels (data not shown). Further study is necessary in order to understand the molecular mechanisms underlying $\beta$ Klotho's involvement in regulation of bile acid synthesis.

The underlying cause of the growth retardation in $\beta k l o t h o^{-/}$mice remains unclear. One might predict that it is also a result of the elevation in bile acid synthesis, although the expression of Cyp7a1 at early stages should be examined.

The results in $\beta k l o t h o^{-/-}$mice revealed the potential of the $\beta$ Klotho system for future medical applications. An increasingly large percentage of the population suffers from cholesterol gallstone formation. Without exhibiting other obvious disease-related phenotypes, $\beta k_{\text {lotho }}-$ - mice produce and excrete higher amounts 
of bile acids and were resistant to cholesterol gallstone formation. Therefore, reagents that interfere with $\beta$ Klotho activity might help to prevent gallstone formation. Also, such reagents may help in the development of novel approaches to facilitating the excretion of lipid-soluble toxins from the body with bile flow.

\section{Methods}

Targeting vector construction and generation of $\beta k_{l o t h 0^{-/-}}$mouse. Genomic DNA clones spanning the $\beta k l o t h o$ locus were isolated from a mouse $129 \mathrm{SVJ}$ genomic library. The $5^{\prime}$ arm of the targeting vector covered a $6.4-\mathrm{kb}$ EcoRI fragment including part of the putative first ATG-containing exon. An 8.0-kb HpaIBam HI fragment was used for the $3^{\prime} \mathrm{arm}$. A promoter-less EGFP gene and a PGKneo cassette were inserted between these 2 arms with the EGFP gene in the forward direction flanked by loxP5171 ( $5^{\prime}$ side) (40) and loxP sequences ( 3 ' side). The PGKneo cassette was in the reverse direction, flanked by loxP sequences. Vector DNA was linearized and introduced into TT2 ES cells via electroporation. G418-resistant clones were selected and screened by Southern blotting. Out of 439 clones, 3 were selected and injected into 8 cell stage Crlj:CD1 (ICR) embryos. Germline transmission occurred in 2 chimeras derived from a clone. Male chimeras were crossed with female C57BL/6 mice, and heterozygous offspring were intercrossed to obtain $\beta$ klotho $\sigma^{-/-}$mice. Offspring from these heterozygous parents were used in this study; however, we confirmed that the same phenotypes were also observed in offspring of heterozygous parents backcrossed 5 times to C57BL/6.

Animals and diets. Mice were housed in plastic colony cages in a temperature-controlled room $\left(24^{\circ} \mathrm{C}\right)$ with a 12 -hour light/12-hour dark cycle under specific pathogen-free conditions, and all experimental procedures were approved by the Committee on Animal Research (Faculty of Medicine, Kyoto University). Standard feed was the NMF diet (Oriental Yeast Co.). The other diets, including the basal diet (no. 5755), 1\% CA diet (no. 58794), 1\% CDCA diet (no. 5T11), and atherogenic diet (no. 5806C-Q), were purchased from PMI Feeds Inc. In metabolic experiments, mice were individually housed in metabolic cages, and stool and urine samples were collected every 24 hours for 3 days. Food, water, stool, and urine were weighed daily.

Antibodies and protein analyses. A portion of the $\beta$ klotho cDNA (corresponding to amino acids 814-967, C-terminal half of the $\beta K L 2$ domain; ref. 1) was cloned into the EcoRI site of the pGEX-4T-1 expression vector (Amersham Biosciences) to produce a partial $\beta$ Klotho-GST fusion protein, which was introduced into E. coli (BL21). After separation by SDS-PAGE, the band corresponding to the partial $\beta$ Klotho-GST fusion protein was excised from the gel and injected into an SD rat purchased from Japan SLC Inc. The splenocytes of the immunized rat were used for the establishment of monoclonal anti- $\beta$ Klotho antibodies after hybridoma selection. Western blotting was performed with diluted anti- $\beta$ Klotho antibody $(1: 2,500)$ against $15 \mu \mathrm{g}$ of total protein.

1. Ito, S., et al. 2000. Molecular cloning and expression analyses of mouse betaklotho, which encodes a novel Klotho family protein. Mech. Dev. 98:115-119.

2. Kuro-o, M., et al. 1997. Mutation of the mouse klotho gene leads to a syndrome resembling ageing. Nature. 390:45-51.

3. McCarter, J.D., and Withers, S.G. 1994. Mechanisms of enzymatic glycoside hydrolysis. Curr. Opin. Struct. Biol. 4:885-892.

4. Davies, G., and Henrissat, B. 1995. Structures and mechanisms of glycosyl hydrolases. Structure. 3:853-859.

5. Kozoni, V., Tsioulias, G., Shiff, S., and Rigas, B. 2000. The effect of lithocholic acid on proliferation and apoptosis during the early stages of colon carcinogenesis: differential effect on apoptosis in the presence of a colon carcinogen. Carcinogenesis.
Northern blot analysis. Total RNAs were extracted using the Total RNA Isolation System (Promega), and $30 \mu \mathrm{g}$ aliquots were subjected to the analysis. Blots were exposed to the imaging plate (Fujifilm) and analyzed by a Storm 860 scanner (Molecular Dynamics). Detection probes were obtained from cDNA fragments amplified by RT-PCR with gene-specific primer pairs. Each fragment was cloned into a pT7Blue T-Vector (Novagen), and sequences were confirmed. After excision from the vector by restriction enzymes, the fragments were labeled by $\alpha^{32} \mathrm{P}-\mathrm{dCTP}$ using a Megaprime DNA Labeling Kit (Amersham Biosciences) and subjected to the hybridization.

Plasma and tissue chemistry. Mice were anesthetized by diethylether, and whole blood was collected from hearts. Serum was separated from collected blood by centrifugation. Serum cholesterol concentrations were determined using Cholesterol E-Test Wako (Wako Pure Chemical Industries). The concentrations of other plasma constituents were determined by SRL Inc. Hepatic total lipids were extracted using Bligh and Dyer's method (41) and resuspended in $2 \%$ Triton X-100 by sonication. Following extraction, hepatic cholesterol levels were determined by using the Cholesterol E-Test Wako (Wako Pure Chemical Industries). Bile acid pool size was determined using Total Bile Acids Test Wako (Wako Pure Chemical Industries) following ethanol extraction from a mixed tissue sample including liver, gallbladder, and whole small intestine (15). Fecal total bile acids were also determined using the same kit after ethanol extraction (15). Fecal cholesterol was determined by Japan Food Research Laboratories using gas chromatography (GC-1700, Shimadzu; and DB-1 column, Agilent).

Statistical analysis. Unless otherwise noted, all values are expressed as the mean \pm SD. All data were analyzed by 2 -tailed, unpaired Student's $t$ test for significant differences between the mean values using Statcel (OMS Publishing Inc.) for Macintosh. $P$ values less than 0.05 were considered to be statistically significant.

\section{Acknowledgments}

We thank T. Obata for technical assistance and R. Yu for helpful comments on the manuscript. This research was partially supported by a Ministry of Education, Science, Sports, and Culture Grantin-Aid to S. Ito and Y.-i. Nabeshima and a grant from the Ministry of Heath, Labour, and Welfare to Y.-i. Nabeshima.

Received for publication August 18, 2004, and accepted in revised form May 31, 2005.

Address correspondence to: Yo-ichi Nabeshima, Department of Pathology and Tumor Biology, Graduate School of Medicine, Kyoto University, Yoshida Konoe-cho, Sakyo-ku, Kyoto 606-8501, Japan. Phone: 81-75-753-4422; Fax: 81-75-753-4676; E-mail: nabemr@lmls.med.kyoto-u.ac.jp.
21:999-1005

6. Staudinger, J.L., et al. 2001. The nuclear receptor PXR is a lithocholic acid sensor that protects against liver toxicity. Proc. Natl. Acad. Sci. U. S. A. 98:3369-3374.

7. Davis, R.A., Miyake, J.H., Hui, T.Y., and Spann, N.J. 2002. Regulation of cholesterol-7alpha-hydroxylase: BAREly missing a SHP [review]. J. Lipid Res. 43:533-543.

8. Russell, D.W. 2003. The enzymes, regulation, and genetics of bile acid synthesis. Annu. Rev. Biochem. 72:137-174.

9. Sinal, C.J., et al. 2000. Targeted disruption of the nuclear receptor FXR/BAR impairs bile acid and lipid homeostasis. Cell. 102:731-744.

10. Marrapodi, M., and Chiang, J.Y. 2000. Peroxisome proliferator-activated receptor alpha (PPARalpha) and agonist inhibit cholesterol 7alpha-hydroxylase gene (CYP7A1) transcription. J. Lipid Res, 41:514-520.

11. Post, S.M., et al. 2001. Fibrates suppress bile acid synthesis via peroxisome proliferator-activated receptor-alpha-mediated downregulation of cholesterol 7alpha-hydroxylase and sterol 27-hydroxylase expression. Arterioscler. Thromb. Vasc. Biol. 21:1840-1845.

12. Chiang, J.Y. 2002. Bile acid regulation of gene expression: roles of nuclear hormone receptors [review]. Endocr. Rev. 23:443-463.

13. Gupta, S., Stravitz, R.T., Dent, P., and Hylemon, P.B. 2001. Down-regulation of cholesterol 7alphahydroxylase (CYP7A1) gene expression by bile acids in primary rat hepatocytes is mediated by the c-Jun $\mathrm{N}$-terminal kinase pathway. J. Biol. Chem. 
276:15816-15822.

14. Stravitz, R.T., Vlahcevic, Z.R., Gurley, E.C., and Hylemon, P.B. 1995. Repression of cholesterol 7 alpha-hydroxylase transcription by bile acids is mediated through protein kinase $\mathrm{C}$ in primary cultures of rat hepatocytes. J. Lipid Res. 36:1359-1369.

15. Yu, C., et al. 2000. Elevated cholesterol metabolism and bile acid synthesis in mice lacking membrane tyrosine kinase receptor FGFR4. J. Biol. Chem. 275:15482-15489.

16. Holt, J.A., et al. 2003. Definition of a novel growth factor-dependent signal cascade for the suppression of bile acid biosynthesis. Genes Dev. 17:1581-1591.

17. Meier, P.J., and Stieger, B. 2002. Bile salt transporters. Annu. Rev. Physiol. 64:635-661.

18. Trauner, M., and Boyer, J.L. 2003. Bile salt transporters: molecular characterization, function, and regulation [review]. Physiol. Rev. 83:633-671.

19. Li-Hawkins, J., et al. 2002. Cholic acid mediates negative feedback regulation of bile acid synthesis in mice. J. Clin. Invest. 110:1191-1200. doi:10.1172/ JCI200216309.

20. Dawson, P.A., et al. 2003. Targeted deletion of the ileal bile acid transporter eliminates enterohepatic cycling of bile acids in mice. J. Biol. Chem 278:33920-33927.

21. Schwarz, M., Russell, D.W., Dietschy, J.M., and Turley, S.D. 2001. Alternate pathways of bile acid synthesis in the cholesterol 7alpha-hydroxylase knockout mouse are not upregulated by either cholesterol or cholestyramine feeding. J. Lipid Res. 42:1594-1603.

22. Kanda, T., et al. 1996. Effect of bile on the intestinal bile-acid binding protein (I-BABP) expression. In vitro and in vivo studies. FEBS Lett. 384:131-134.

23. Miyake, J.H., et al. 2002. Transgenic expression of cholesterol-7-alpha-hydroxylase prevents atherosclerosis in C57BL/6J mice. Arterioscler. Thromb. Vasc. Biol. 22:121-126.

24. Ko, C.W., and Lee, S.P. 1999. Gallstone formation. Local factors [review]. Gastroenterol. Clin. North Am. 28:99-115.

25. Dam, H., and Hegardt, F.G. 1971. The relation between formation of gallstones rich in cholesterol and the solubility of cholesterol in aqueous solutions of bile salts and lecithin. Z. Ernabrungswiss. 10:239-252.

26. Van Erpecum, K.J., and Van Berge-Henegouwen, G.P. 1999. Gallstones: an intestinal disease? [review]. Gut. 44:435-438.

27. Leuschner, U. 1992. Oral bile acid treatment of biliary cholesterol stones. Recenti. Prog. Med. 83:392-399.

28. Yu, L., et al. 2002. Disruption of Abcg5 and Abcg8 in mice reveals their crucial role in biliary cholesterol secretion. Proc. Natl. Acad. Sci. U. S. A. 99:16237-16242.

29. Goldstein, J.L., and Brown, M.S. 1990. Regulation of the mevalonate pathway. Nature. 343:425-430.

30. Goodwin, B., et al. 2000. A regulatory cascade of the nuclear receptors FXR, SHP-1, and LRH-1 represses bile acid biosynthesis. Mol. Cell. 6:517-526.

31. Schoonjans, K., and Auwerx, J. 2002. A sharper image of SHP. Nat. Med. 8:789-791.

32. Brendel, C., Schoonjans, K., Botrugno, O.A. Treuter, E., and Auwerx, J. 2002. The small heterodimer partner interacts with the liver $\mathrm{X}$ receptor alpha and represses its transcriptional activity. Mol.
Endocrinol. 16:2065-2076.

33. Wang, L., et al. 2002. Redundant pathways for negative feedback regulation of bile acid production. Dev. Cell. 2:721-731.

34. Kerr, T.A., et al. 2002. Loss of nuclear receptor SHP impairs but does not eliminate negative feedback regulation of bile acid synthesis. Dev. Cell. 2:713-720.

35. Wang, L., Han, Y., Kim, C.S., Lee, Y.K., and Moore, D.D. 2003. Resistance of SHP-null mice to bile acid-induced liver damage. J. Biol. Chem. 278:44475-44481.

36. Yu, C., Wang, F., Jin, C., Huang, X., and McKeehan, W.L. 2005. Independent repression of bile acid synthesis and activation of c-Jun $\mathrm{N}$-terminal kinase (JNK) by activated hepatocyte fibroblast growth factor receptor 4 (FGFR4) and bile acids. J. Biol. Chem. 280:17707-17714.

37. Peet, D.J., et al. 1998. Cholesterol and bile acid metabolism are impaired in mice lacking the nuclear oxysterol receptor LXR alpha. Cell. 93:693-704.

38. Xu, G., et al. 2003. FXR-mediated down-regulation of CYP7A1 dominates LXRalpha in longterm cholesterol-fed NZW rabbits. J. Lipid Res. 44:1956-1962.

39. Repa, J.J., et al. 2002. Regulation of ATP-binding cassette sterol transporters ABCG5 and ABCG8 by the liver X receptors alpha and beta. J. Biol. Chem. 277:18793-18800.

40. Lee, G., and Saito, I. 1998. Role of nucleotide sequences of loxP spacer region in Cre-mediated recombination. Gene. 216:55-65.

41. Bligh, E.G., and Dyer, W.J. 1959. A rapid method of total lipid extraction and purification. Can. J. Biochem. Biophys. 37:911-917. 